Historic, Archive Document

Do not assume content reflects current scientific knowledge, policies, or practices. 



\section{ANCV 91915}

\section{PUYALLUP \\ NURSERY}

\section{A. LINGHAM, Proprietor \{PRICE LIST\} SEASON 1914---1915}

1 year, 4 to 6 feet

2 years, 6 to 8 feet

;) years, selected

\section{}

\section{APPLES}

each

$20 \mathrm{c}$

$25 \mathrm{c}$

$35 \mathrm{c}$

\section{CHERRIES}

1 year, 4 to 6 feet (selected)

2 years, 5 to 7 feet

3 year's, 6 to $\&$ feet, selected

- $35 \mathrm{c}$

- $50 \mathrm{c}$

- $75 \mathrm{c}$

PEARS

1 year, 4 to 6 feet

$30 \mathrm{c}$

2 years, 5 to 7 feet

3 years, 6 to 8 feet, heavy

$40 \mathrm{c}$

$50 \mathrm{c}$

\section{PLUMS AND PRUNES}

1 year, 4 to 6 feet - - - - $30 \mathrm{c}$

2 years, 6 to $\&$ feet - - $\quad 40 \mathrm{c}$

3 years, 6 to 8 feet, heavy

$50 . \mathrm{c}$

\section{PEACHES AND APRICOTS}

1 year, 4 to 6 feet

$25 \mathrm{c}$

2 years, 5 to 7 feet

$35 \mathrm{c}$

\section{QUINCES}

1 year, 3 to 4 feet

2 years, 5 to 7 feet

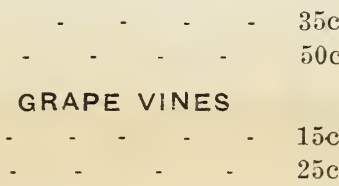

\section{CURRANTS}

1 year Fays Prolific (red)

2 years, 2 to 3 feet, Fays Prolific (red)

Perfection Currant, 1 year

\section{GOOSEBERRIES}

Champion, 1 year

Joselyn and New Sorts

$25 \mathrm{c}$

$\$ 1.50$

$\$ 10.00$

2.50

15.00

\section{$\$ .75$}

1.00

$\$ 5.00$

$10 c$

$15 \mathrm{c}$

1.50

6.00

10.00

\section{BLACKBERRIES AND HYBRID SORTS}

Snyder, Kittatinny

each per doz

$\begin{array}{rrr}5 c & \$ .50 & \$ 3.00 \\ 10 \mathrm{c} & .75 & 4.00 \\ 10 \mathrm{c} & .75 & 4.00 \\ 15 \mathrm{c} & 1.50 & 10.00\end{array}$

Lawton, Eldorado -

Ner'sereau

Ifimalaya Giant
$10 \cdot c$

$\$ 1.00$

$\$ 6.00$

1.50

10.00
$\$ 25.00$

35.00

40.00

$\$ 20.00$

30.00

$\$ 30.00$

40.00

$\$ 5.00 \quad 40.00$

$\$ 15.00$
20.00
30.00

30.00

$5.00 \quad 45.00$

25.00

35.00

(1)




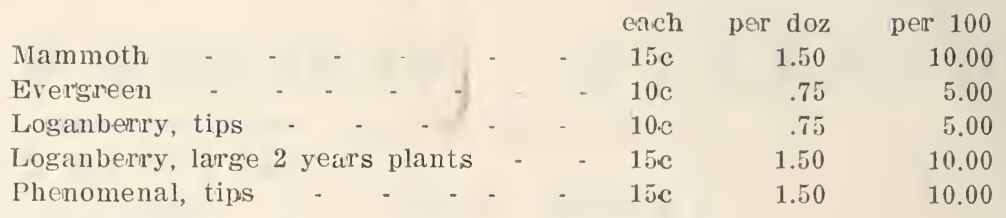

\section{RASPBERRIES}

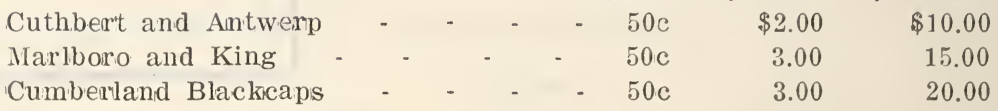

\section{STRAWBERRIES}

Marshall, Magoon, etc - - - - $25 \mathrm{c}$

Alpine Everbearing - - $\quad$ - $\quad$ - $35 \mathrm{c}$

$\$ .75 \$ 5.00$

$1.50 \quad 10.00$

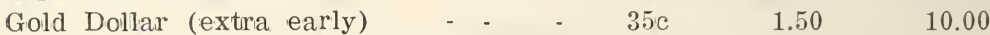

Goodell Strawberry (something exura good) 35c $\quad 1.50$

Write for special prices per thousand on Raspiberries and small fruit plants.

\section{RHUBARB}

1 year roots, Victoria Mammoth - $\$ .75$

2 year" roots - $\quad$ - $\quad$ - $\quad$ - $\quad$ - 1.00

Selected, divided roots - $\quad-\quad-1.50$

ASPARAGUS

1 year roots

2 year roots

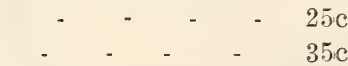

\section{HEDGE PLANTS}

California Privet, 1 year

California Privet, 2 years

Japan Barberry, 18 to 24 in

Veronica (box-leaf dwarf for edging borders) per 100

$\$ 5.00$

6.00

10.00

$\$ 1.00$

1.50

$\$ 8.00$

10.00

$\$ 4.00$

$\$ 30.00$

5.00

10.00

40.00

75.00

5.00

\section{VINES}

Clematis, large flowering, 2 yr.. (Jackmani, Henryi, etc.)

each

per doz

per 100

$\$ 5.00$

3.00

3.50

3.50

Honeysuckles, 2 and 3 years

25 to $35 \mathrm{c}$

35 to $50 \mathrm{c}$

35 to $50 \mathrm{c}$

ROSES, 100 LEADING SORTS

2 years, Medium - $\quad$ - $\quad$ - $\quad$ - $25 \mathrm{c}$

2 years, select - $\quad$ - $\quad$ - $\quad$ - $\quad$ - $35 c$

3 years, heavy - $\quad-\quad-\quad-\quad-50 \mathrm{c}$

New Sorts - $\quad$ - $\quad$ - $\quad 35$ to $75 \mathrm{c}$

Tree Roses - $\quad$ - $\quad$ - $\quad$. $\quad$ - $\$ 1.00$

$\$ 3.00 \quad \$ 20.00$

$3.50 \quad 25.00$

$4.50 \quad 35.00$

FLOWERING SHRUBS

Althea (Rose of Sharon)

Almond-dbl. flowering

Barberry, purple 


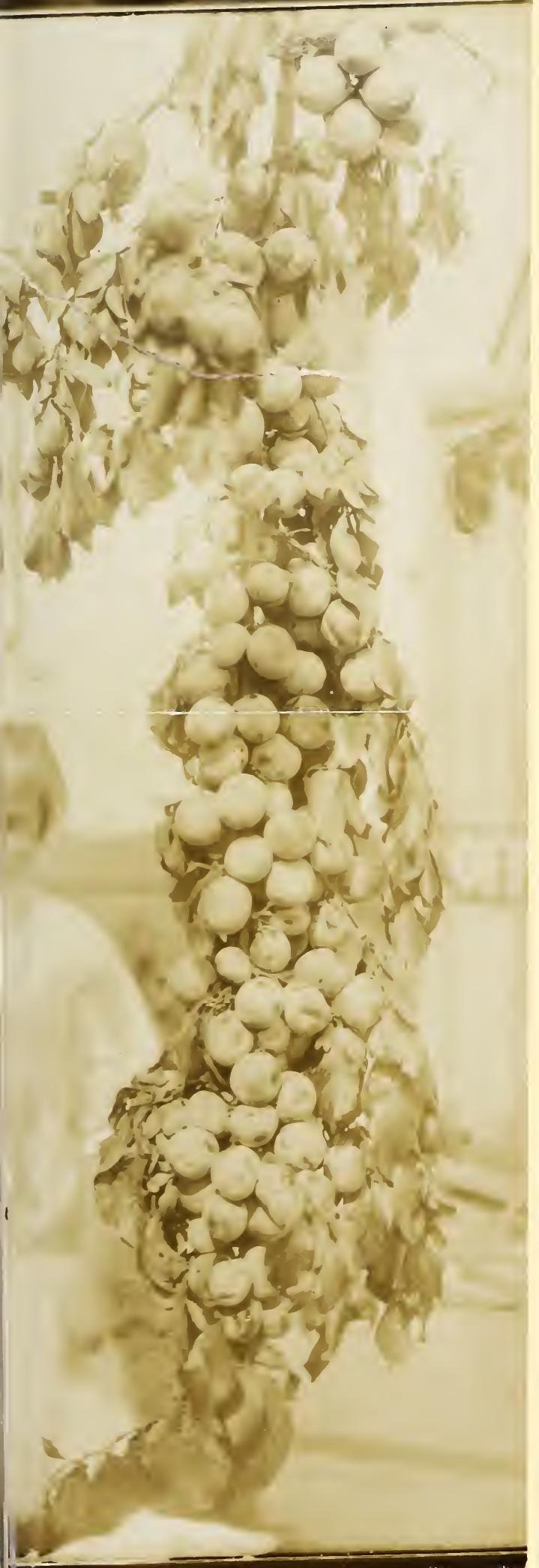


PUYYALLUP NURSERY

Photograph taken ofep I 15 . subject "Satje Alum." Thuit boine on one himb: Tree pilanted in fall of 1912 then $2 \mathrm{~g}$. Ld. Grown by Q. Dingham. Auralluf Tasis. 


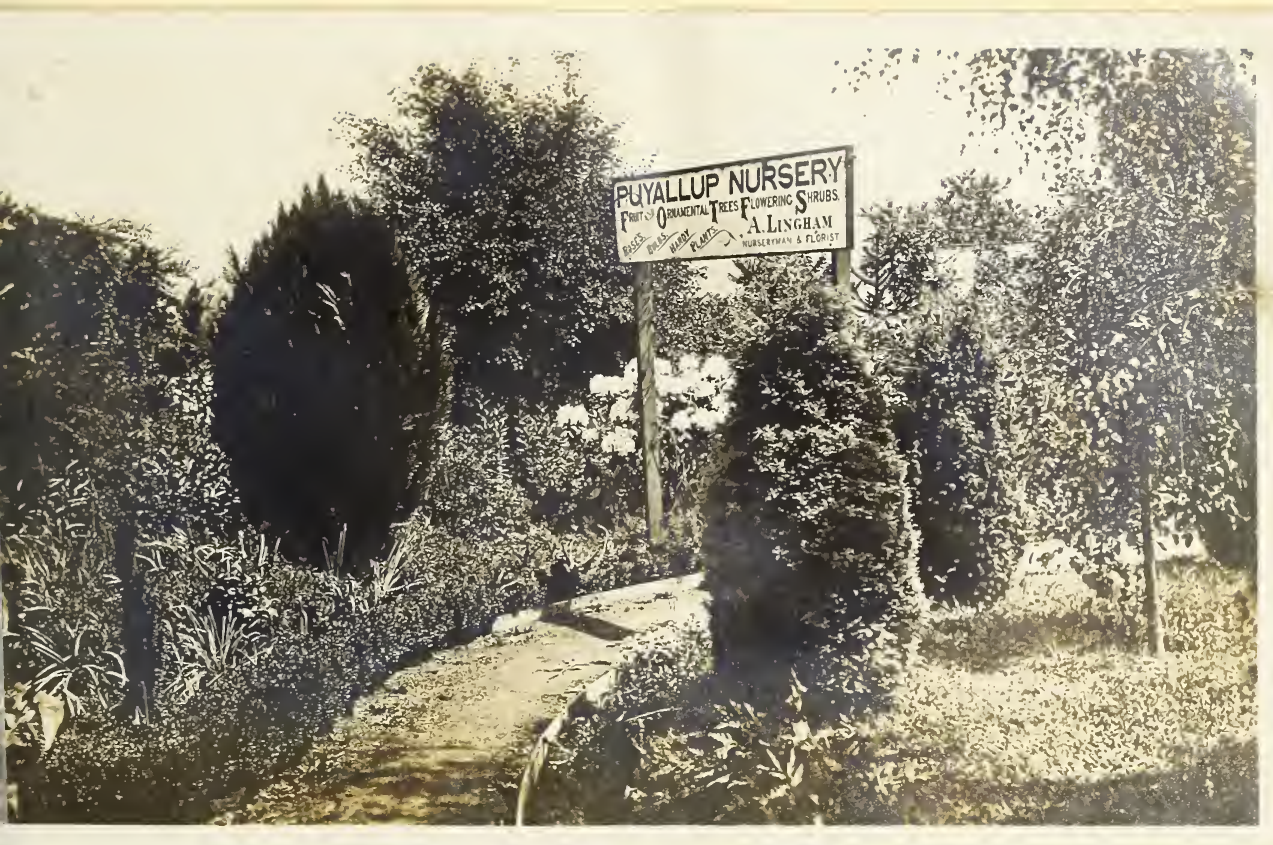




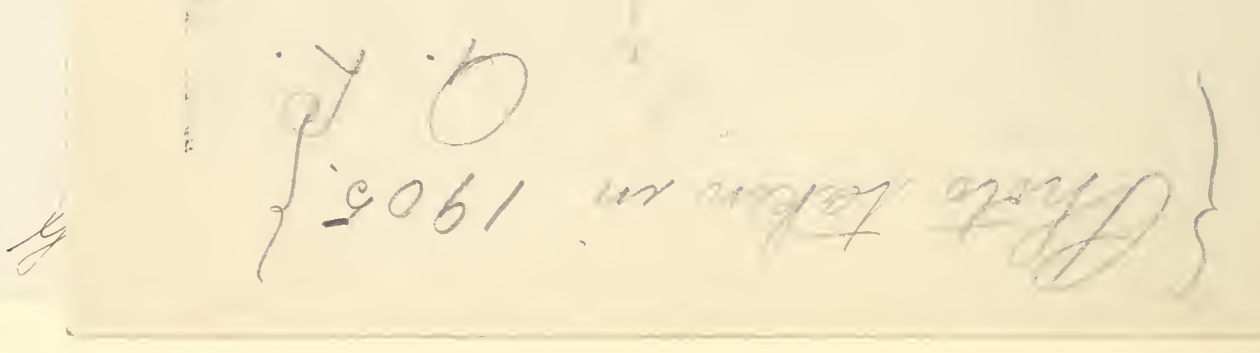




Barberry Japan
Calycanthus

\section{PEONIES}

Common sorts $\quad-\quad$ - $\quad$ - $\quad .25$ to $\$ .50$

Fancy named - $\quad$ - $\quad$ -.$\tau$ to 1.00

HARDY PERENNIALS AND MISCELLANEOUS

An?mone (Japan hardy) clumps - - $25 \mathrm{c}$

Delphinum Belladonna (turquois blue) 20 c

Delphinum formosa (deep blue) - - $15 \mathrm{c}$

Iris, German assorted - $\quad$ - $\quad$ - $10 \mathrm{c}$

2.00

15.00

Iris, Japanese, assorted - _ - $\quad$ - 20c

1.50

10.00

Hardy Pinks (double white) - _ . $10 \mathrm{c}$

1.00

8.00

Tampas grass, clumps - . $\quad 50 \mathrm{c}$

Pampas grass, plants - $\quad$ - $\quad$ - $25 \mathrm{c}$

Carnation plants - - $\quad$ - . $10 \mathrm{c}$

2.00

15.00

Gypsophila paniculata - - - - 20c

Gypsophila paniculata, double flig.. - $\quad 25 \mathrm{c}$

Stoke's Aster plants, 15c, Clumps - $25 \mathrm{c}$

Foxglove clumps $\quad$ - $\quad$ - $\quad$ - $\quad$ - $\quad$ - $10 \mathrm{c}$

1.00

4.50

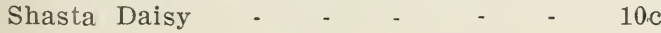

Bamboo (small clumps) - - - $\quad$ - 50c

Golden Glow $\quad$ - $\quad$ - $\quad$ - $\quad$ - $\quad$ - $\quad 15 \mathrm{c}$

Double sunflower $\quad-\quad$ - $\quad$ - $\quad$ - $\quad 20 \mathrm{c}$

Hollyhocks - $\quad$ - $\quad$ - $\quad$ - $\quad$ - $15 \mathrm{c}$

Zebra grass - $\quad$ - $\quad$ - $\quad$ - $\quad$ - $20 \mathrm{c}$

Phlox, perennial, 6 colors - - - $\quad 20 \mathrm{c}$

Primrose, hardy sorts - - - - $\quad 10 \mathrm{c}$

Pansy plants, in bloom - - - $\quad 5 \mathrm{c}$

Violets, single and double - - - 5 c

Gladiolus bulbs, fancy mixed - - $5 \mathrm{c}$

Gladiolus fancy named sorts

10 to $25 \mathrm{c}$

Easter Lily bulbs - $\quad$ - $\quad$ - $15 \mathrm{c}$

Auratum Lily bulbs

$\$ 15.00$

Cactus Dahlias, tubers

$20 \mathrm{c}$

Decorative and fancy quilled

$20 \mathrm{c}$

Dahlias, rooted plants .

$20 \mathrm{c}$ 
NUT, SHADE AND ORNAMENTAL TREES

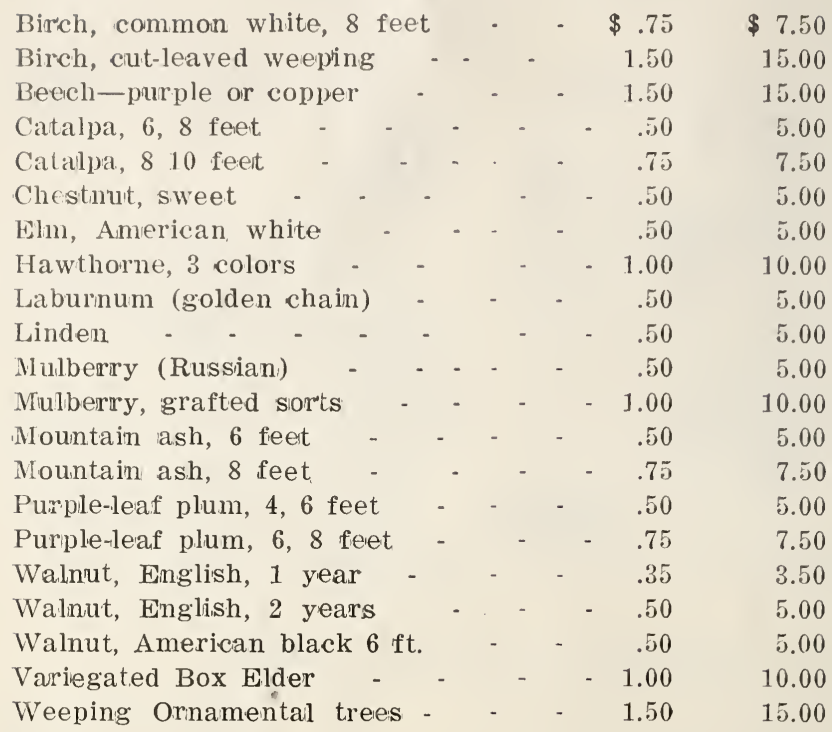

HARDY EVERGREEN SHRUBS AND TREES

Araucaria (Monkey puzzle tree) $\$ 2.00$ to $\$ 4.00$

Cypress (Lawson's - $\quad-\quad-\quad .50$ to 1.00

Cypress, dwarf erect, $1 \frac{1 / 2}{2}$ to 2 fieet $\quad 1.00$

Cypress dwarf, 3 feet specimens $\quad 2.00$

Cypress Japan sorts - - 1.00 to 2.00

Spruce, Koster's blue, grafted 2.00

Cottoneaster, assorted - $\quad-.35$ to .50

Boxwood, speciments - $\quad .50$ to 1.00

Mountain Laurel - - $\quad 1.00$ to 1.50

Magnolia grandiflora - - $\quad 1.50$

Rhododendron seedings - $\quad 1.00 \quad 10.00$

Rhododendron grafted named sorts $\quad 2.00 \quad 20.00$

Holly English grafted, (with berries) 1.00 each foot

Holly variegated sorts - - $\quad 1.50$ each foot

Yuccas, assorted - $\quad$ - $\quad .50$ to 1.00

Junipers, pyramidal - $\quad$ - $\quad .50$ each foot

Yews, English (specimens) - $\quad .50$ to $\$ 1.00$ ear'h

Yews, Irish (plain and golden) .75 each foot

\section{HARDY BULBS FOR FALL PLANTING}

Crocus, assorited colors - $15 \mathrm{c}$ per doz, 1.00 per 100

Daffodils, fancy trumpet sorts ramed $\quad .50$ per dcz.

Jonquils (fragiant flowers) .35 per doz.

Narcissus, "Poets' single or double white .35 per doz.

Hyacinths, assorted colors - $\quad .75$ to 1.00 per doz.

Tulips, assorted - - - $\quad .35$ per doz.

Tulips Darwin and late sorts $\quad .50$ per doz. 